\title{
Sudarshan and modern quantum optics
}

\author{
G. S. Agarwal* \\ Institute for Quantum Science and Engineering and Department of Biological and Agricultural Engineering, Texas A\&M University, \\ College Station, Texas 77843, USA and Department of Physics and Astronomy, Texas A\&M University, College Station, Texas 77843, USA
}

I dedicate this short article to the memory of E. C. G. Sudarshan. I especially like to recall the great contributions that he made to quantum optics when the field was very much in infancy. Discussing physics with him was always a great learning experience for me as he would provide new insights into a subject. To my mind he was one with a phenomenal knowledge of physics and mathematics. Over the years I had many discussions on a variety of subjects which were dear to me - these included optical coherence $^{1}$; quantum Zeno effect ${ }^{2}$, theory of open quantum systems $s^{3,4}$, weak measurements ${ }^{5}$, higher order poles of S matrix ${ }^{6-8}$ and Pancharatnam-Berry phase ${ }^{9}$. Sudarshan made outstanding contributions to each of these subjects. It was also remarkable that he would suggest experiments on abstract ideas, some of which were successfully carried out by others. For example, his proposal ${ }^{5}$ for realizing weak measurements via optical elements was implemented by Hulet and collaborators ${ }^{10}$. The impact of Sudarshan's work is evident now in the above-mentioned subjects. These are extensively followed by experimentalists as it is becoming more and more possible to implement ideas and thought experiments due to progress in experimental techniques. Before I discuss his work on the diagonal coherent state representation, I give a few examples which clearly show that his work is leaving a mark. His work on quantum Zeno effect ${ }^{2}$ is important in controlling decoherence, which is especially a big problem in quantum computation. There are many laboratory demonstrations on how using short pulse sequences, one can stop evolution due to interaction with the environment ${ }^{11-16}$. The weak measurements using optical experiments with pre- and post-selection of polarization of light are now becoming increasingly popular. For example, it is possible to do a full tomography of a higher dimensional state of a light beam ${ }^{17}$. Another of his classic works was on open systems. Sudarshan, Gorini, and Kossakowsky derived the most general form of the Liouvillian for an open quantum system which would preserve positivity. Unfortunately for reasons unknown, this structure is known as the Lindblad form. It might be worth noting that the two works appeared at the same time, though Sudarshan's paper was submitted a little earlier. This rigorous structure of the master equation is important as the approximations or phenomenological descriptions can make the density matrix negative.

\footnotetext{
*e-mail: girish.agarwal@tamu.edu
}

I would like to discuss now, Sudarshan's discovery of what he called diagonal coherent state representation. The paper was received by the Physical Review Letters on 1 March 1963, and published on 1 April 1963. It has been cited more than 1000 times, which is quite a record for a theoretical paper. The paper was published with a rather unusual title 'Equivalence of semiclassical and quantum mechanical descriptions of statistical light beams'. The key result of the paper was that every density matrix

$$
\rho=\sum_{n, n^{\prime}=0}^{\infty} \rho\left(n, n^{\prime}\right)|n\rangle\left\langle n^{\prime}\right|
$$

can be represented in the diagonal form

$$
\rho=\iint_{-\infty}^{\infty} \mathrm{d}^{2} z \phi(z)|z\rangle\langle z|
$$

where $|z\rangle$ is a coherent state and the function $\phi$ is related to the elements of the density matrix via

$$
\phi(z)=\sum_{n, n^{\prime}=0}^{\infty} \rho_{n, n^{\prime}}(-1)^{n+n^{\prime}} \mathrm{e}^{|z|^{2}} \frac{1}{\sqrt{n ! n^{\prime} !}} \frac{\partial^{n+n^{\prime}}}{\partial z^{n} \partial z^{* n^{\prime}}} \delta^{(2)}(z)
$$

I have expressed it in a form in which it is more often used now. Using this form he shows that 'classical complex representations can be put in one-to-one correspondence with quantum mechanical density matrices'. He realizes that the function $\phi$ is not necessarily positive definite. He then generalizes the diagonal representation to countable number of degrees of freedom and states. 'Consequently the description of statistical states of a quantum mechanical system with arbitrary (countably infinite) number of degrees of freedom is completely equivalent to the descriptions in terms of the classical probability distributions in the same number (countably infinite) of complex variables.' In the early days these statements have been taken literally ${ }^{18}$, as they stand to imply that quantum treatment will yield the same results as classical treatment. Clearly this cannot be the case in general. It is clear from the form of $\phi$ in the paper (eq. (6) there) that for a Fock state $|l\rangle$ of the radiation field, the function $\phi$ is highly singular.

$$
\rho\left(n, n^{\prime}\right)=\delta_{n n^{\prime}} \delta_{n l},
$$




$$
\phi(z)=\exp \left(|z|^{2}\right) \frac{1}{l !} \frac{\partial^{2 l}}{\partial z^{l} \partial z^{* l}} \delta^{(2)}(z)
$$

and thus cannot be classical, i.e., $\phi(z)$ does not have the characteristic features of a classical probability distribution.

Sudarshan himself clarified the situation after the publication of the paper in his lectures and elsewhere. In the book Fundamentals of Quantum Optics by Klauder and Sudarshan $^{19}$, which is based on the lectures of Sudarshan, it is clearly stated on p. 192 that 'relations which are formally similar to the classical calculations ... this similarity in form does not carry any connotation that the quantum theory is physically equivalent to the classical theory, but only that it can be set up to have a formal equivalence. It is in this sense that the "optical equivalence theorem" has been formulated and should be interpreted in the general sense.' Sudarshan's derivation of diagonal coherent representation applies to arbitrary density matrices of the radiation field both diagonal and nondiagonal, whereas earlier work derives such a representation for the Bose-Einstein distribution of the radiation field ${ }^{20}$. Glauber called his representation as $P$ representation and with the passage of time, the diagonal coherent state representation and the $P$ representation are referred to as Glauber-Sudarshan representation.

The modern developments in quantum optics and quantum information science depend on our ability to produce states with strict quantum properties, and one needs quantitative measures for characterizing such quantumness. Besides, the measures must be within experimental reach. That is where the great utility of the diagonal coherent state representation lies. This is because the detectors measure quantities which are the mean values of the normally ordered operators involving the field operators. Further as Sudarshan showed, these normally ordered expectation values are simply related to the averages of classical moments over the function $\phi(z)$. Thus all the measures for quantumness have been developed based on the fact that the function $\phi(z)$ could be negative and singular. The most popular among these measures include those for phase related nonclassicality - squeezing parameter S; moment-related nonclassicality-Mandel Q parameter. A variety of other measures were also developed to characterize situations when both Q and S do not work. A whole host of measures can be found for example in my book ${ }^{21}$. The field of developing newer measures for quantumness based on Sudarshan's $\phi(z)$ continues to be active. It may be noted that the emerging field of quantum sensing is based on the ability to produce quantum states and characterize the quantumness of such states. Many nonclassical states were discovered in the decades following Sudarshan's work-some of the important ones are squeezed states, macroscopic superpositions of coherent states, photon-added coherent and thermal states. In all such cases, the function $\phi$ is very far from a classical distribution and this is a strong signature of the nonclassicality of such states ${ }^{21}$. The great quantum revolution that is to take place in this century would depend on our ability to produce and manipulate quantum states, especially single-photon states. This is where we will see increasing importance of Sudarshan's work on diagonal representation.

It was a great privilege for me to know him very well and to have had many opportunities for discussions with him on a variety of topics.

1. Sudarshan, E. C. G., Equivalence of semiclassical and quantum mechanical descriptions of statistical light beams. Phys. Rev. Lett., 1963, 10, 277.

2. Misra, B. and Sudarshan, E. C. G., The Zenos paradox in quantum theory. J. Math. Phys., 1977, 18, 756.

3. Sudarshan, E. C. G., Mathews, P. M. and Rau, J., Stochastic dynamics of quantum-mechanical systems. Phys. Rev., 1961, 121, 920.

4. Gorini, V., Kossakowski, A. and Sudarshan, E. C. G., Completely positive dynamical semigroups of Nlevel systems. J. Math. Phys., 1976, 17, 821 .

5. Duck, I. M., Stevenson, P. M. and Sudarshan, E. C. G., The sense in which a 'weak measurement' of a spin particle's spin component yields a value 100. Phys. Rev. D, 1989, 40, 2112.

6. Sudarshan, E. C. G., Quantum dynamics in dual spaces. Phys. Rev. A, 1994, 50, 2006

7. Chiu, C. B., Sudarshan, E. C. G. and Bhamathi, G., Cascade model: A solvable field theory. Phys. Rev. D, 1992, 46, 3508.

8. Bhamathi, G. and Sudarshan, E. C. G., Double resonances and Jordan block spectra. Int. J. Mod. Phys. B, 1996, 10, 1531.

9. Simon, R., Kimble, H. J. and Sudarshan, E. C. G., Evolving geometric phase and its dynamical manifestation as a frequency shift: an optical experiment. Phys. Rev. Lett., 1988, 61, 19.

10. Ritchie, N. W. M., Story, J. G. and Hulet, R. G., Realization of a measurement of a weak value. Phys. Rev. Lett., 1991, 66, 1107.

11. Viola, L. and Lloyd, S., Dynamical suppression of decoherence in two-state quantum systems. Phys. Rev. A, 1998, 58, 2733.

12. Agarwal, G. S., Scully, M. O. and Walther, H., Inhibition of decoherence due to decay in a continuum. Phys. Rev. Lett., 2001, 86, 4271 .

13. Khodjasteh, K. and Lidar, D. A., Fault-tolerant quantum dynamical decoupling. Phys. Rev. Lett., 2005, 95, 180501.

14. Du, J., Rong, X., Zhao, N., Wang, Y., Yang, J. and Liu, R. B., Preserving electron spin coherence in solids by optimal dynamical decoupling. Nature, 2009, 461, 1265 EP.

15. Biercuk, M. J., Uys, H., VanDevender, A. P., Shiga, N., Itano, W. M. and Bollinger, J. J., Optimized dynamical decoupling in a model quantum memory. Nature, 2009, 458, 996 EP.

16. Cao, Y. et al., Direct counterfactual communication via quantum Zeno effect. Proc. Natl. Acad. Sci., 2017, 114, 4920.

17. Shi, Z., Mirhosseini, M., Margiewicz, J., Malik, M., Rivera, F., Zhu, Z. and Boyd, R. W., Scan-free direct measurement of an extremely high-dimensional photonic state. Optica, 2015, 2, 388.

18. Glauber, R. J., Coherent and incoherent states of the radiation field. Phys. Rev., 1963, 131, 2766 (see especially p. 2788).

19. Klauder, J. R. and Sudarshan, E. C. G., Fundamentals of Quantum Optics, Dover Publications, 2006, p. 192.

20. Glauber, R. J., Photon correlations. Phys. Rev. Lett., 1963, 10, 84.

21. Agarwal, G. S., Quantum Optics, Cambridge University Press, 2013.

doi: $10.18520 / \mathrm{cs} / \mathrm{v} 116 / \mathrm{i} 2 / 205-206$ 